\title{
Apancreatic pigs cloned using Pdx1-disrupted fibroblasts created via TALEN-mediated mutagenesis
}

\author{
Jin-Dan Kang ${ }^{1, *}$, Hyojin Kim ${ }^{2,4,}{ }^{*}$, Long Jin ${ }^{1, *}$, Qing Guo ${ }^{1}$, Cheng-Du Cui ${ }^{1}$, Wen-Xue Li ${ }^{1}$, \\ Seokjoong Kim ${ }^{3}$, Jin-Soo Kim² and Xi-Jun Yin ${ }^{1}$ \\ ${ }^{1}$ Jilin Provincial Key Laboratory of Transgenic Animal and Embryo Engineering and Department of Animal Science, Yanbian \\ University, Yanji 133002, China \\ ${ }^{2}$ Center for Genome Engineering, Institute for Basic Science, Gwanak-gu, Seoul 151-747, South Korea \\ ${ }^{3}$ ToolGen, Inc., Byucksan Kyoungin Digital Valley 2-Cha, Geumcheon-Gu, Seoul 153-023, South Korea \\ ${ }^{4}$ Present/Current address: Department of Biosystems Science and Engineering, ETH Zurich, Basel CH-4058, Switzerland \\ *These authors have contributed equally to this work \\ Correspondence to: Jin-Soo Kim, email: jskim01@snu.ac.kr \\ Xi-Jun Yin, email: yinxj33@msn.com
}

Keywords: PDX1; TALEN; SCNT; pancreas; pig

Received: September 30, $2017 \quad$ Accepted: December 05, $2017 \quad$ Published: December 14, 2017

Copyright: Kang et al. This is an open-access article distributed under the terms of the Creative Commons Attribution License 3.0 (CC BY 3.0), which permits unrestricted use, distribution, and reproduction in any medium, provided the original author and source are credited.

\section{ABSTRACT}

Pancreatic and duodenal homeobox 1 (PDX1) plays a crucial role in pancreas development, $\beta$-cell differentiation, and maintenance of mature $\beta$-cell function. In this study, we designed a strategy to produce PDX1-knockout (KO) pigs. A transcription activator-like effector nuclease (TALEN) pair targeting exon 1 of the swine PDX1 gene was constructed. Porcine fetal fibroblasts (PFFs) were transfected with the TALEN plasmids plus a surrogate reporter plasmid. PDX1-mutated PFFs were enriched by magnetic separation and used to produce homozygous PDX1-KO pigs via a twostep somatic cell nuclear transfer (SCNT) cloning process. In the first SCNT step, we obtained eight fetuses, established PFF cell lines, and analyzed PDX1 gene mutations by T7 endonuclease 1 assays and Sanger sequencing. Five fetuses showed mutations at the PDX1 loci with two biallelic mutations and three monoallelic mutations (mutation rate of $62.5 \%$ ). In the second step, a PDX1 biallelic mutant PFF cell line with a 2 bp deletion in one allele and a 4 bp insertion in the other allele was used as a donor to generate cloned pigs via SCNT. From 462 cloned embryos transferred into two surrogates, nine live piglets were delivered. These piglets at birth were not clearly distinguishable phenotypically from wild-type piglets, but soon developed severe diarrhea and vomiting and all died within 2 days after birth. Dissection of PDX1-KO piglets revealed that the liver, gallbladder, spleen, stomach, common bile duct, and other viscera were present and normal, but the pancreas was absent in all cases.

\section{INTRODUCTION}

Pancreatic and duodenal homeobox 1 (PDX1), a transcription factor identified a decade ago during a search for insulin transcription regulatory mechanisms, plays a critical role in an array of pancreatic and islet functions [1]. The critical role of PDX1 in pancreatic development was first demonstrated in the Pdx1-knockout (KO) mouse model $\left(\mathrm{Pdx}^{-/-}\right)$in which pancreatic agenesis is observed, coupled with a failure of the pancreatic bud to grow [2]. Naturally occurring non-functional mutations in the PDX1 gene have been reported in people born with pancreatic agenesis [3-6]. One pancreatic agenesis patient initially presented with hyperglycemia. Despite aggressive dietary and insulin therapy, she failed to gain weight in the first 18 days of life. She was homozygous for a point deletion 
in the PDX1 gene. The patient received insulin and pancreatic enzyme replacement therapy and developed normally [4].

Considering the closer similarity of humans with pigs than with mice in terms of anatomy, physiology, and the genome, pigs can serve as a large animal model to study human metabolism and physiology [7, 8]. Given that various mutations in the human PDX1 gene give rise to a spectrum of disease phenotypes, ranging from the complete absence of the pancreas to maturity onset diabetes of the young [4, 9], pigs with PDX1 gene mutations would be a useful animal model to study the association of diabetes mellitus and human pancreatic agenesis diseases. Transcription activator-like effector nuclease (TALEN)-mediated genome modification has been successfully applied to generate animals with monoallelic or biallelic gene modifications, such as mice [10], pigs [11], and even monkeys [12]. Therefore, the objective of this study was to produce PDX $1^{-/}$pigs using a combination of TALEN technology and somatic cell nuclear transfer (SCNT).

We propose that these animals are useful resources to study human pancreatic agenesis and to develop novel drugs such as pancreatic enzyme replacement therapies. In addition, PDX1-KO pig cells and human induced pluripotent stem cells can be used to generate human pancreases in pigs via blastocyst complementation, which will open up new avenues for organ transplantation.

\section{RESULTS}

\section{Validation of PDX1-targeting TALEN activity and TALEN-driven genome editing}

We designed a TALEN pair tartgeting to transactivation domain in exon 1 of the swine PDX1 gene (Figure 1A). The activity of the PDX1-TALEN was evaluated using a surrogate reporter system. In these assays, the induction of small indel mutations in the TALEN target sequence upstream of the inactive eGFP and $\mathrm{H}-2 \mathrm{~K}^{\mathrm{k}}$ cell surface reporter genes can restore the expression of these genes, while mRFP is constitutively expressed independent of TALEN activity. Primary porcine fetal fibroblasts were transformed with PDX1targeting TALEN and surrogate reporter plasmids. Cells expressing eGFP were detected only upon co-transfection of TALEN and reporter plasmids, validating the activity of the PDX1-TALEN in primary porcine fetal fibroblasts. Incubation of transfected cells with magnetic beads (MACSelect $\mathrm{K}^{\mathrm{k}}$ microbeads; Miltenyi Biotech, Germany) conjugated to an anti-H-2 $\mathrm{K}^{\mathrm{k}}$ antibody and elution through a MACS LS column (Miltenyi Biotech) led to the separation of $\mathrm{H}-2 \mathrm{~K}^{\mathrm{k}}$-expressing cells $[13,14]$. These $\mathrm{H}-2 \mathrm{~K}^{\mathrm{k}}$-positive cells were enriched with eGFP-expressing cells (Figure 1B).
Additionally, to directly evaluate the extent of TALEN-induced mutations, genomic DNA isolated from sorted and unsorted cell populations was subjected to T7E1 assays and Sanger sequencing. PCR amplified a $251 \mathrm{bp}$ band, whereas PCR products from cells transformed with the PDX1-TALEN showed a cleaved band upon T7E1 digestion, confirming the presence of mutations in the transformed cells. The PDX1 mutation frequency was higher in magnetically sorted cells than in unsorted cells (36\% vs. $11 \%$, Figure 1C). 6 out of 10 clones harbored mutations at the PDX1-TALEN-tarted site (60\%) and two of them harbored 141bp large deletion in PDX1 gene (Figure 1D).

\section{Production of PDX1-KO fetuses by SCNT}

PFFs collected from females were transfected with the PDX1-TALENs and reporter plasmids by electroporation, and transfected cells were enriched magnetically as described previously [11]. H-2K $\mathrm{K}^{\mathrm{k}}$-positive cells expressing both mRFP and eGFP (Figure 1B) were cultured for two additional days and were used as donor cells in SCNT. The competency of reconstructed embryos cultured in vitro was investigated by examining the development of blastocysts. The blastocyst development rate $(18.1 \%$ vs. $19.5 \%$, Figure 2$)$ and the mean number of cells per blastocyst $(38.7 \pm 8.1$ vs. $39.7 \pm 4.2)$ were similar in embryos reconstructed with transformed and non-transformed donor cells (Table 1). To produce PDX1mutant fetuses, 386 embryos cloned using $\mathrm{H}-2 \mathrm{~K}^{\mathrm{k}}$-positive PFFs were transferred into two surrogate mothers (R725 and R801). Eight fetuses were surgically collected from R-725 after 26 days of gestation, and two fetuses (Figure 3 A) were surgically collected from R-801 after 29 days of gestation (Table 2).

\section{Detection of mutations and genotyping of PDX1- mutant fetuses}

Fibroblast cell lines from the eight fetuses were analyzed individually by T7E1 assays and by sequencing PCR products covering the target locus. Mutations at the target locus (PDX1 gene) were present in five of the eight fetuses (62.5\%; Table 2). Two fetuses (F1 and F2) carried biallelic mutations and three fetuses (F3, F4, and F5) carried monoallelic mutations in the PDX1 gene. Among the fetuses with biallelic PDX1 mutations, fetus F1 had a $2 \mathrm{bp}$ deletion in one allele and a $4 \mathrm{bp}$ insertion in the other allele, whereas fetus F2 had a 30 bp deletion in one allele and a $3 \mathrm{bp}$ insertion in the other allele (Figure 3B).

\section{Generation of biallelic PDX1-KO piglets by SCNT}

PFFs were established from fetus F1, which carried a $2 \mathrm{bp}$ deletion in one allele and a $4 \mathrm{bp}$ insertion in the 
other allele, and were used as nuclear donors to produce $\mathrm{PDX}^{-/-}$piglets by SCNT. A total of 462 cloned embryos were transferred to two surrogates in estrus, resulting in the delivery of nine live piglets (Table 3 ).

Newborn PDX1/- piglets were comparable to their WT littermates. However, within the first day postpartum, $\mathrm{PDX} 1^{-/}$animals show signs of growth retardation and dehydration, although they fed because their stomachs contained milk (Figure 4). By 1 day postpartum, the stomachs of some $\mathrm{PDX}^{-/-}$piglets were distended due to a lack of gastric emptying into the gut
(Figure 4C). The serum glucose level was undetectable in these PDX1-null piglets or lower than in WT piglets, and PDX1-null animals showed signs of growth retardation within the first day postpartum (Table 4). These pups often suffered from severe diarrhea and vomiting, and died 2 days after birth. The developmental retardation of PDX1-null pups is likely attributable to malnutrition resulting from a lack of digestion in the absence of the pancreas and functional rostral duodenum and/or diabetic consequences due to the absence of the pancreas. Dissection of PDX1-null pups revealed that

(A)

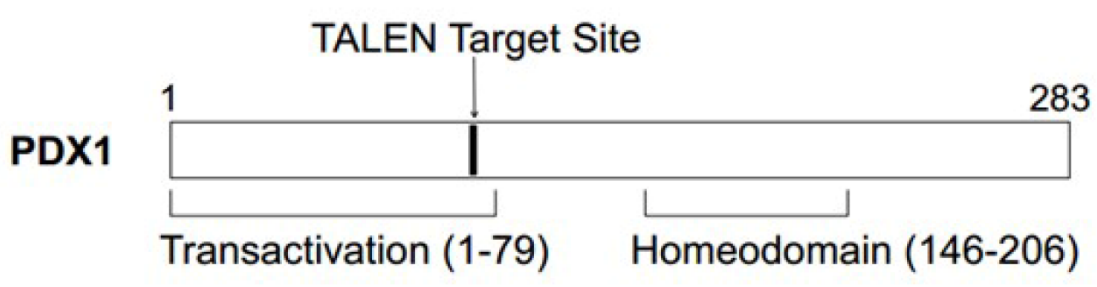

(B)
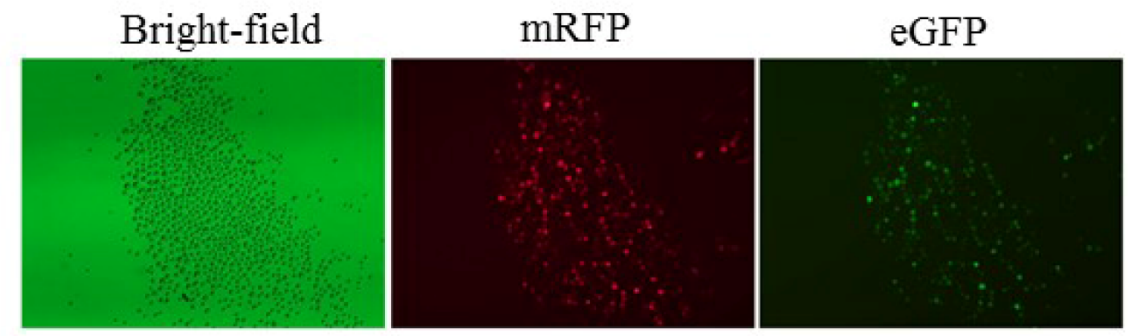

(C)

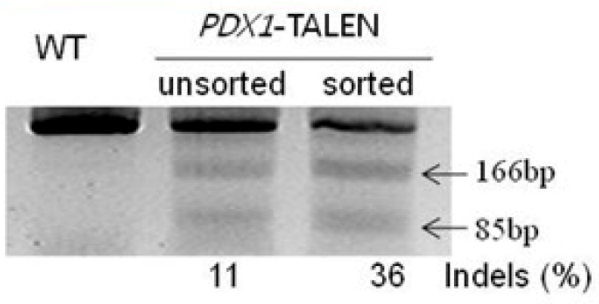

(D) Sorted $60 \%(=6 / 10)$

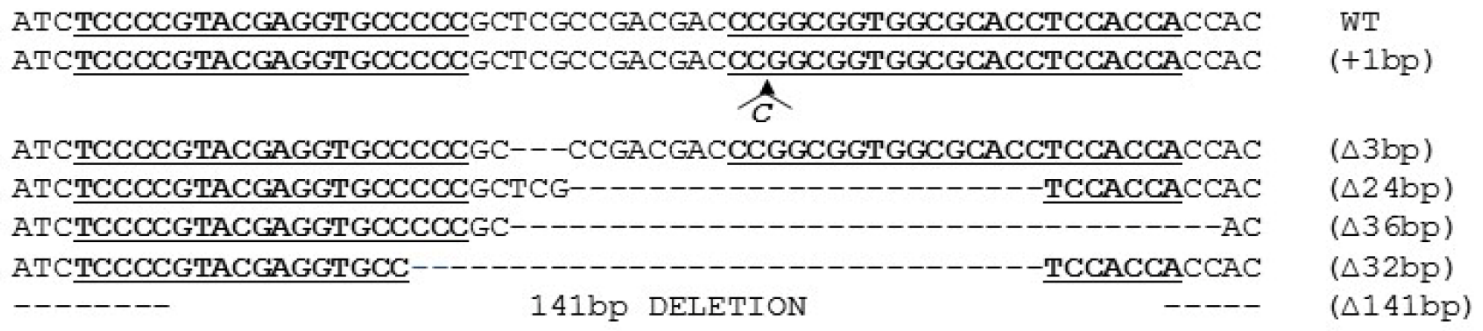

Figure 1: Generation of $P D X 1$-disrupted PFFs using PDX1-TALEN. (A) Schematic representation of functional domains of $P D X 1$ and the PDX1-TALEN target site. (B) Magnetic separation-mediated enrichment of PDX1-disrupted PFFs. PFFs were cotransfected with $P D X 1$-targeting TALENs and reporter plasmids, followed by magnetic separation of $\mathrm{H}$ - $2 \mathrm{~K}^{\mathrm{k}}$-expressing cells designed to express both mRFP and eGFP. Fluorescence microscopy examination was performed 1 day after magnetic separation. Magnification, 40×. PDX1TALEN-driven mutations at endogenous chromosomal sites in magnetic separated PFFs. (C) The T7E1 assay were used to detect PDX1TALEN-driven mutations. Arrows indicate the expected positions of DNA bands cleaved by T7E1. Mutation frequencies (Indels (\%)) were calculated by measuring the band intensities). (D) DNA sequences of the PDX1 wild-type (WT) and mutant clones. The region of the target sequence of the PDX1-TALEN is shown in underlined, deleted bases are indicated by dashes. The column on the right indicates the number of inserted or deleted bases. 
the liver, gallbladder, spleen, stomach, common bile duct, and other viscera were present and normal, but the pancreas was noticeably absent (Figure 4). Histological results suggested that the main organs of $\mathrm{PDX} 1^{-/-}$piglets displayed no abnormalities (Figure 5).

\section{DISCUSSION}

One of the cost-effective way is routinely used to generate genetically-modified small animals is directed injection of the gene targeting nucleases into zygote, including rat [15], mice [16], and rabbit [17]. However, many of the resulted founder animals were chimeric ones with multiple mutations through the embryo injection [17]. To get the animals with a single mutation, one or two more round of further breeding have to be employed for selection among the offspring. Clearly, this method is not suitable for large animals, such as pigs, have long gestation cycles, high recipient costs, which make it difficult to achieve large animal offspring with a single expected mutation. SCNT has been successfully applied to generate $\mathrm{KO}$ animals in species lacking germline transmitting embryonic stem cells, including pigs, sheep and cattle. In this study, we successfully generated PDX $1^{-/}$ fetus by specifically targeting the exon 1 site of porcine PDX1 gene with TALEN technology and somatic cell nucleus transfer.

First for SCNT, the fibroblast cell lines were established from one fetus, expanded, and cultured. Cells at an early passage were preserved in liquid nitrogen. Magnetically separated PFFs were used in SCNT, and the results indicated that the competence of reconstructed embryos derived from magnetically separated PFFs did not markedly differ from that of untreated embryos, with a similar rate of blastocyst development and a similar number of cells per blastocyst. Furthermore, pregnancies were maintained by both surrogates implanted with reconstructed embryos derived from magnetically separated PFFs, while other studies reported surrogate pregnancy rates of 36-90\% [18-21]. Taken together, our results indicate that surrogate reporter-based cell enrichment is effective for selecting nuclear donor cells with nuclease-mediated gene modification.

To generate $\mathrm{PDX}^{-/}$piglets, we used a two-step SCNT method (recloning), in which PFFs derived from the first SCNT were used as nuclear donors for the second SCNT. This recloning method very efficiently generates animals, especially livestock, with targeted gene mutations $[22,23]$. Compared with clonally selected transformed fibroblasts, fetal fibroblasts derived from the first SCNT have some advantages for the generation of gene-edited animals. Selection of nuclear donor cells with genetic modifications frequently involves drug treatment of cells in long-term culture. These cultures are inevitably at greater risk of cell exhaustion and senescence [24], which are associated with chromosomal aberrations [25]. Immediately after birth, $\mathrm{PDX}^{-/}$piglets were indistinguishable from their WT littermates. However, within the first day postpartum, $\mathrm{PDX}^{-/}$animals show signs of growth retardation and dehydration, although they fed because their stomachs contained milk. By 1 day postpartum, the stomachs of some $\mathrm{PDX} 1^{-/}$piglets were distended due to a lack of gastric emptying into the gut. The serum glucose level was undetectable in these PDX1null piglets or lower than in WT piglets, and PDX1-null animals showed signs of growth retardation within the first day postpartum. These pups often suffered from severe

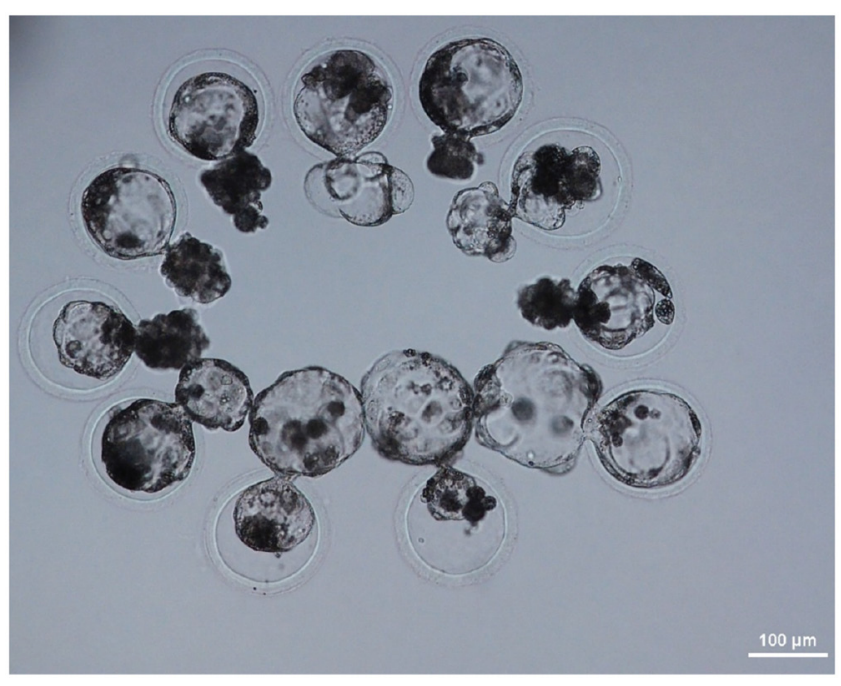

Figure 2: Day 7 SCNT blastocysts derived from PFFs transfected with PDX1-TALEN plasmids. 
Table 1: In vitro development of reconstructed pig embryos with donor PFFs transfected with or without PDX1TALEN plasmids

\begin{tabular}{lcccc}
\hline Donor cell type & $\begin{array}{c}\text { No. of } \\
\text { embryos }\end{array}$ & $\begin{array}{c}\text { No. of } \\
\text { 2-4-cell embryos (\%) }\end{array}$ & $\begin{array}{c}\text { No. of } \\
\text { blastocysts (\%) }\end{array}$ & $\begin{array}{c}\text { No. of cells per blastocyst } \\
\text { (mean } \pm \text { SEM) }\end{array}$ \\
\hline Non-transfected PFF & 232 & $204(88.0)$ & $42(18.1)$ & $38.7 \pm 8.1$ \\
Transfected PFF & 261 & $222(85.1)$ & $51(19.5)$ & $39.7 \pm 4.2$ \\
\hline
\end{tabular}

diarrhea and vomiting, and died 2 days after birth. The developmental retardation of PDX1-null pups is likely attributable to malnutrition resulting from a lack of digestion in the absence of the pancreas and functional rostral duodenum and/or diabetic consequences due to the absence of the pancreas [2]. Dissection of PDX1-null pups revealed that the liver, gallbladder, spleen, stomach, common bile duct, and other viscera were present and normal, but the pancreas was noticeably absent, reminiscent of pancreatic agenesis in Pdx1-null mice [2] and human patients [4]. Histological results suggested that the main organs of $\mathrm{PDX}^{-/}$piglets displayed no abnormalities.

With unparalleled recent progress in the field of pluripotent stem cells and genome editing, an elegant interspecies chimeric complementation approach is on the horizon to generate functional human organs using animals as hosts [26]. Rat pancreases have been

(A)
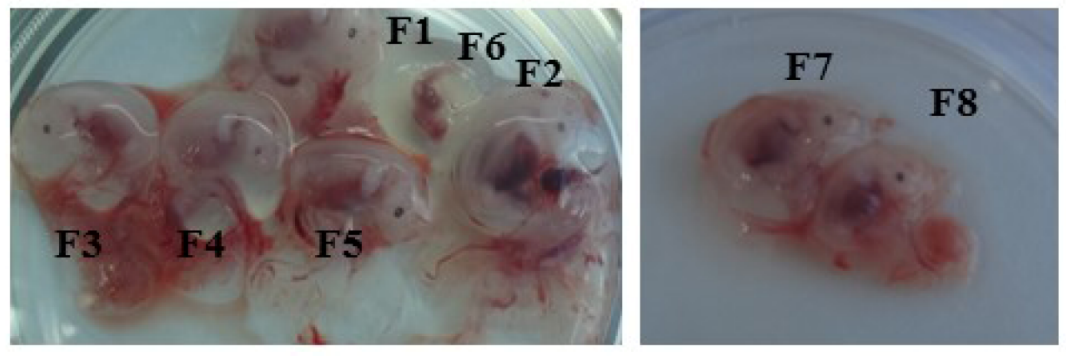

(B)

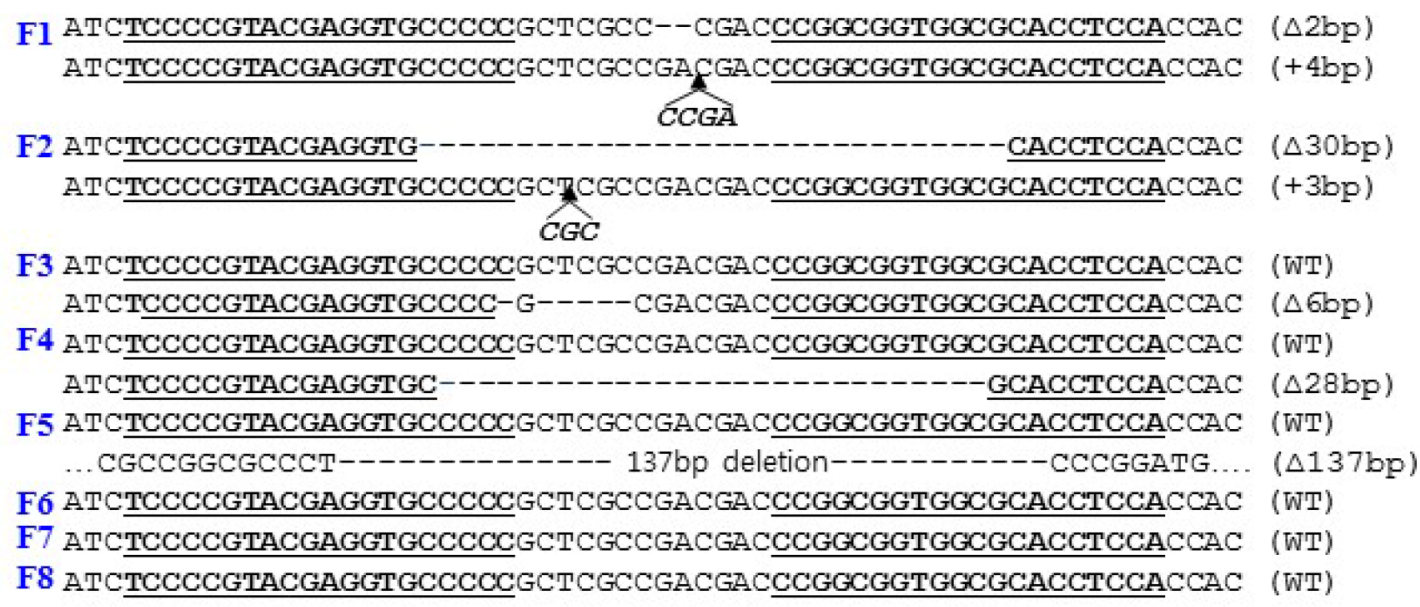

Figure 3: Photographs of the fetuses and PDX1 sequences. (A) Fetuses derived from TALEN-treated PFFs at day 26 (left) and day 29 (right). (B) PDX1 sequences from TALEN-treated fetuses. DNA sequences of the WT and mutant clones, with TALEN recognition sites underlined, deleted bases indicated by dashes, and inserted bases in italics. Two of eight fetuses harbored biallelic mutations and three harbored monoallelic mutations in PDXI. 
Table 2: Summary of the production of $P D X 1$-KO fetuses using $P D X 1$-TALEN and SCNT

\begin{tabular}{lcccccc}
\hline Recipient & $\begin{array}{c}\text { No. of embryos } \\
\text { transferred }\end{array}$ & $\begin{array}{c}\text { No. of } \\
\text { fetuses }\end{array}$ & $\begin{array}{c}\text { Days of } \\
\text { gestation }\end{array}$ & $\begin{array}{c}\text { Fetal fibroblast } \\
\text { cell lines }\end{array}$ & $\begin{array}{c}\text { Mono-allelic cell } \\
\text { lines (\%) }\end{array}$ & $\begin{array}{c}\text { Bi-allelic cell } \\
\text { lines (\%) }\end{array}$ \\
\hline R725 & 205 & 6 & 26 & $\begin{array}{c}\text { F1, F2, F3, } \\
\text { R8, F5, F6 }\end{array}$ & $\begin{array}{c}\text { F3, F4, F5 } \\
(50.0)\end{array}$ & $\begin{array}{c}\text { F1, F2 } \\
(33.3)\end{array}$ \\
R801 & 181 & 2 & 29 & F7, F8 & - & $(0 \%)$ \\
\hline
\end{tabular}

Table 3: Production of biallelic PDX1-KO pigs by SCNT

\begin{tabular}{lccc}
\hline Surrogate number & No. of embryos transferred & No. of live offspring & Information about the piglets \\
\hline R115 & 208 & $3(\mathrm{RC} 1-3)$ & Diarrhea and vomiting \\
& & & All piglets died at day 2 \\
\hline R118 & 254 & $6(\mathrm{RC} 4-9)$ & Diarrhea and vomiting \\
& & Two piglets died at day 1 \\
\hline
\end{tabular}

A
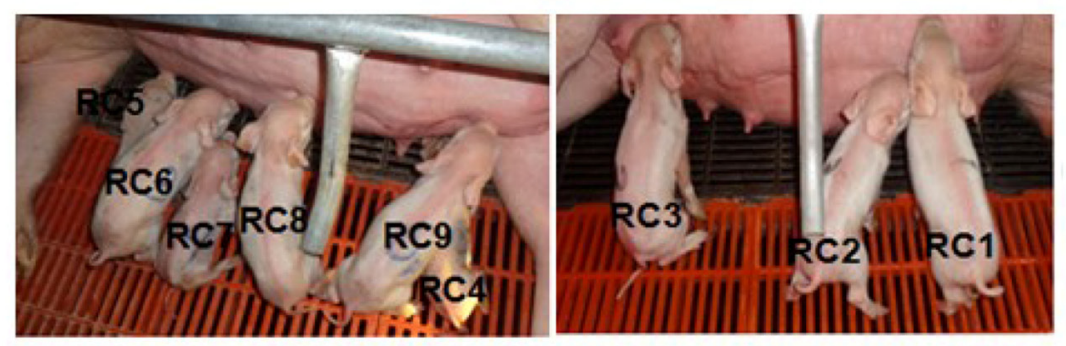

B

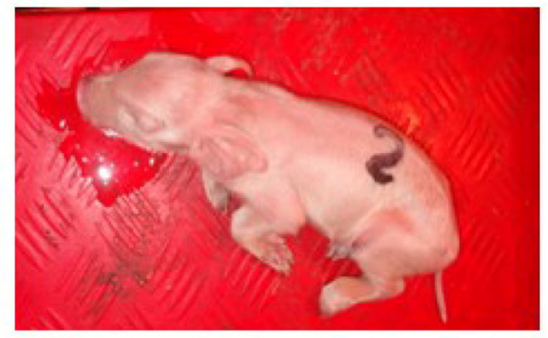

$\mathrm{C}$
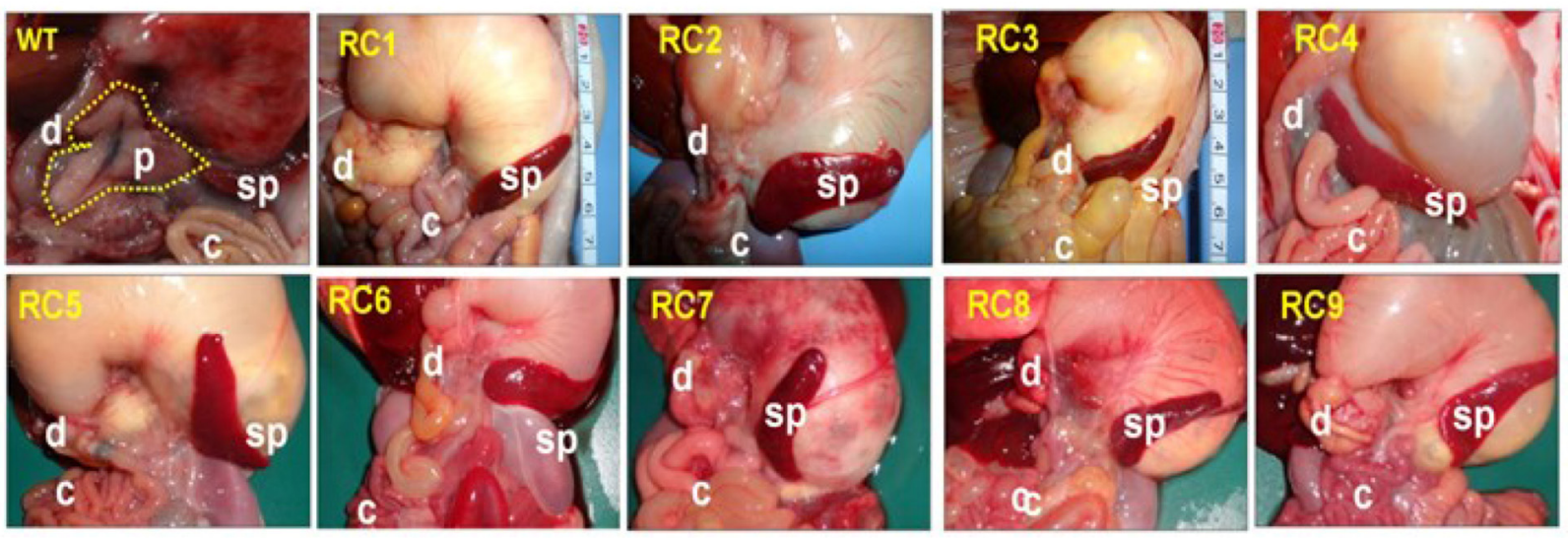

Figure 4: PDXI-KO piglets. (A) Newborn $P D X 1^{-/}$piglets were delivered from two recipients. (B) $P D X 1^{-/}$piglets suffered from extreme vomiting before death. (C) Analysis of pancreatic development in WT and biallelic mutant pups (RC1-9). A WT piglet had a normal pancreas (dotted line). Faithful reproduction of the pancreatogenesis-disabled phenotype was observed in nine $P D X 1^{-\digamma}$ piglets (RC1-9). s, stomach; p, pancreas; d, duodenum; sp, spleen; c, colon. 
Table 4: Serum glucose concentrations at 1 day after birth and pancreatic phenotype of $P D X 1$-KO and WT pigs

\begin{tabular}{lcccc}
\hline \multirow{2}{*}{ Piglet } & Blood glucose $(\mathbf{m m o l} / \mathbf{L})^{*}$ & \multicolumn{2}{c}{ Body weight $\mathbf{( k g )}$} & \multirow{2}{*}{ Pancreatogenesis } \\
\cline { 3 - 4 } & Low & 1-day-old & 2-day-old & None \\
RC1 & Low & 1.3 & $1.2(\mathrm{died})$ & None \\
RC3 & Low & 1.3 & $1.1(\mathrm{died})$ & None \\
RC4 & Low & 1.0 & $0.9(\mathrm{died})$ & None \\
RC5 & Low & $0.5(\mathrm{died})$ & - & None \\
RC6 & 2.1 & 1.0 & $0.9(\mathrm{died})$ & None \\
RC7 & 1.7 & 1.0 & $1.0(\mathrm{died})$ & None \\
RC8 & Low & 0.7 & $0.7(\mathrm{died})$ & None \\
RC9 & 1.2 & $0.8(\mathrm{died})$ & - & None \\
\hline WT1 & 4.6 & 0.9 & $0.9(\mathrm{died})$ & Normal \\
WT2 & 3.3 & 1.2 & 1.4 & Normal \\
WT3 & 3.6 & 1.1 & 1.2 & Normal \\
\hline
\end{tabular}

*Blood glucose levels were measured at 1 day after birth. Low indicates the blood glucose level was lower than $0.6 \mathrm{mmol} / \mathrm{L}$.

successfully generated in $\mathrm{Pdx}^{-/}$mice by combining the principle of blastocyst complementation with the production of interspecific chimeras [27]. A recent report showed that an intermediate type of human induced pluripotent stem cells (hiPSCs) exhibits a higher degree of chimerism and can generate differentiated progenies in post-implantation pig embryos [28]. Therefore, PDX1$\mathrm{KO}$ pigs and hiPSCs can be used to generate human pancreases in pigs via blastocyst complementation, a method that will open up new avenues for organ transplantation [29].
In summary, we used a PDX1-specific TALEN to create frameshift mutations in PFFs, which were subsequently used to generate PDX1 biallelic mutant piglets via SCNT. These piglets at birth were not clearly distinguishable phenotypically from 1-day-old WT piglets, but soon developed severe diarrhea and vomiting and all died within 2 days after birth. Dissection of PDX1$\mathrm{KO}$ piglets revealed that the pancreas was absent in all cases. This would be a useful animal model to study the association of human pancreatic agenesis and diabetes mellitus.

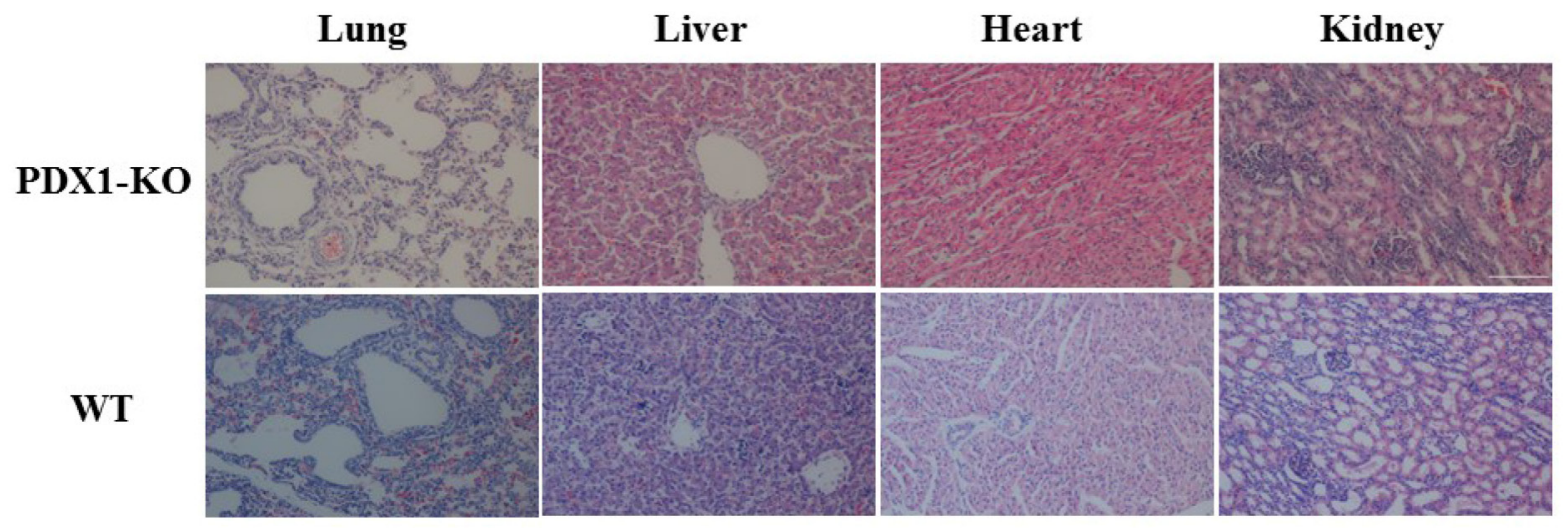

Figure 5: Histological analysis of the main organs from PDX1-KO and WT piglets. Magnification, 200×. Scale bar indicates $100 \mu \mathrm{m}$. 


\begin{tabular}{lll}
\hline & Direction & Sequence (5' to 3') \\
\hline First & Forward & ctgcgtgcctgtacatgggcc \\
& Reverse & Gcgtgagccttggtagactt \\
Second & Forward & ccgcegcetccgttcg \\
& Reverse & gcgtgagccttggtagactt \\
\hline
\end{tabular}

\section{MATERIALS AND METHODS}

\section{Animals and ethics statement}

The protocols of this study were approved by the Committee on the Ethics of Animal Experiments at Yanbian University (Yanji, China). All procedures were performed in strict accordance with the Guide for the Care and Use of Laboratory Animals. All surgical procedures were performed under anesthesia and stringent efforts were made to minimize animal suffering.

\section{TALEN design and construction}

TALEN expression vectors composed of TALEN repeat domains and a FokI nuclease domain containing a Sharkey RR or Sharkey DAS heterodimer mutation for the chosen target sequence were prepared via GoldenGate assembly as described previously [13]. Plasmids harboring the PDX1-targeting TALEN (PDX1-TALEN) were also prepared as described previously [13]. An episomal surrogate reporter containing the chosen target sequence was constructed. TALEN-mediated mutation cells were separated using a column (MACS LS column; Miltenyi Biotech, Germany) according to the manufacturer's instructions [13]. Plasmids encoding PDX1-TALENs and the surrogate reporter were obtained from ToolGen (Seoul, South Korea).

\section{Cell culture and transfection}

Porcine fetal fibroblasts (PFFs) were established from a fetus at day 32 postconception as previously described [30] and cultured for seven passages in Dulbecco's modified Eagle medium (DMEM) supplemented with 1\% nonessential amino acids, $100 \mathrm{U} /$ $\mathrm{mL}$ penicillin, $100 \mathrm{mg} / \mathrm{mL}$ streptomycin, and $15 \%$ fetal bovine serum (FBS). Cells were nucleofected (Amaxa4D, Lonza) with plasmids at a ratio of 45:45:10 (plasmid encoding a TALEN monomer: plasmid encoding the other TALEN monomer: surrogate reporter). A total of $1 \times 10^{6}$ cells was trypsinized using EDTA-Trypsin (WelGen, Seoul, South Korea), washed with HBSS (WelGen, Seoul, South Korea), centrifuged, and finally suspended in $100 \mu \mathrm{L}$ supplied buffer containing TALEN and reporter plasmids. Transfected cells were incubated in humidified 95\% air with $5 \% \mathrm{CO}_{2}$ at $37^{\circ} \mathrm{C}$ for $2 \mathrm{~d}$. The expression of fluorescent proteins was assayed $48 \mathrm{~h}$ later by flow cytometry. Mutant cells were enriched via magnetic separation as described in a previous study [30]. Fibroblast cells were established from a fetus carrying biallelic mutations and cultured in DMEM supplemented with 15\% (v/v) FBS. Cells between passages four and eight were used as donors for nuclear transfer. A single cell suspension was prepared by trypsinization immediately before nuclear transfer.

\section{Generation of fetuses and offspring by SCNT}

Nuclear transfer was performed as described previously [30]. Briefly, mature eggs showing the first polar body were cultured for $1 \mathrm{~h}$ in medium supplemented with $0.4 \mathrm{mg} / \mathrm{mL}$ demecolcine and $0.05 \mathrm{M}$ sucrose, with sucrose added to enlarge the perivitelline space of the eggs. Treated eggs with a protruding membrane were transferred to medium containing $5 \mathrm{mg} / \mathrm{mL}$ cytochalasin $\mathrm{B}$ (CB) and $0.4 \mathrm{mg} / \mathrm{mL}$ demecolcine, and the protrusions were removed with a beveled pipette. A single donor PFF was injected into the perivitelline space of each egg and the cells were electrically fused using two direct current pulses of $150 \mathrm{~V} / \mathrm{mm}$ for $50 \mu \mathrm{s}$ each in $0.28 \mathrm{M}$ mannitol supplemented with $0.1 \mathrm{mM} \mathrm{MgSO}_{4}$ and $0.01 \%$ polyvinyl alcohol. The fused eggs were cultured in NCSU-37 medium for $1 \mathrm{~h}$ before electro-activation. These cells were subsequently cultured in $5 \mathrm{mg} / \mathrm{mL} \mathrm{CB}$-supplemented medium for $4 \mathrm{~h}$, followed by activation of the fused eggs by two direct current pulses of $100 \mathrm{~V} / \mathrm{mm}$ for $20 \mu$ s each in $0.28 \mathrm{M}$ mannitol supplemented with $0.1 \mathrm{mM} \mathrm{MgSO}_{4}$ and $0.05 \mathrm{mM} \mathrm{CaCl}_{2}$. Activated eggs were cultured in medium for 7 days in an atmosphere of 5\% $\mathrm{CO}_{2}$ and $95 \%$ air at $39^{\circ} \mathrm{C}$. Cleavage and blastocyst formation were evaluated on Day 2 and 7, respectively. Cloned embryos at the 1-cell stage after fusion or at the 2-4-cell stage after 1 day of culture were transferred into the oviducts of naturally cycling gilts on the first day of standing estrus. Recipient pigs that received the first SCNT embryos were euthanized at day 26-36 of gestation, and the fetuses were collected. These fetuses were used to confirm the PDX1 mutations.

\section{Detection of mutation and genotyping}

T7E1 assays were performed as described previously [31]. Briefly, genomic DNA was isolated 
using DNeasy Blood \& Tissue Kits (Qiagen, Germany), according to the manufacturer's instructions. The region of DNA containing the TALEN target site was PCR-amplified using the primers listed in Table 5. The amplicons were denatured by heating and annealed to form heteroduplex DNA, which was treated with 5 units of T7E1 (New England Biolabs) for $20 \mathrm{~min}$ at $37^{\circ} \mathrm{C}$ and electrophoresed on agarose gels. To confirm the mutation introduced by TALEN to the target allele, PCR amplicons spanning the target sites were purified using a Gel Extraction Kit (Macherey-Nagel, Germany) and cloned into the T-Blunt vector using the T-Blunt PCR Cloning Kit (SolGent, Daejeon, Korea). The cloned inserts were again amplified using the same primers and sequenced using the M13 primer.

\section{Generation of $\mathrm{PDX1}^{--}$piglets and analysis of blood glucose levels}

PDX1-KO fetuses were used to generate rejuvenated PFFs for the second round of SCNT to produce boars. Pregnancy was assessed ultrasonographically on day 25 . Cloned piglets were delivered naturally or by inducing labor via intramuscular injections of prostaglandin F2 $\alpha$ (Ningbo, China) on day 113 of gestation. Blood samples were subsequently obtained by ear marginal veins, and blood glucose levels were measured using a YUYUETM_710 (Jiangsu, China) handheld blood glucose meter.

\section{Histological analysis}

Tissue samples from wild-type (WT) and PDX1- ${ }^{-1}$ pigs were fixed in $10 \%$ formalin for $24 \mathrm{~h}$ at room temperature and dehydrated in an automated tissue processor. The tissues were embedded in paraffin, sectioned at a thickness of $5 \mu \mathrm{m}$, deparaffinized, rehydrated, and stained with hematoxylin for $5 \mathrm{~min}$. The sections were rinsed in running tap water and stained with eosin for $4 \mathrm{~min}$. Hematoxylin and eosin-stained sections were dehydrated and mounted. Images were captured using a Leica DM5000B microscope.

\section{Statistical analysis}

Data are presented as the mean $\pm \mathrm{SD}$ and are derived from at least three independent experiments. Groups were compared using the Student's t test, with P values $<0.05$ considered statistically significant.

\section{Abbreviations}

PDX1: Pancreatic and duodenal homeobox 1; PDX1KO: PDX1-knockout; TALEN: Transcription activator-like effector nuclease; SCNT: Somatic cell nuclear transfer; PFFs: Porcine fetal fibroblasts; WT: Wild-type.

\section{Author contributions}

Xi-Jun Yin and Jin-Soo Kim designed the study. Jin-Dan Kang, Hyojin Kim, Long Jin, Qing Guo, ChengDu Cui, Wen-Xue Li, Seokjoong Kim and Xi-Jun Yin performed the experiments. Xi-Jun Yin, Jin-Dan Kang, and Hyojin Kim analyzed the data and wrote the manuscript.

\section{ACKNOWLEDGMENTS}

The authors would like to thank the Hanji and Longxing pig farms for providing surrogate pig recipients and their enthusiasm and support for this research.

\section{CONFLICTS OF INTEREST}

The authors declare that they have no competing interests.

\section{FUNDING}

This work was supported by the Institute for Basic Science in South Korea (Grant No. IBS-R021-D12015-a02) and the State Key Development Program for Basic Research of China (Grant No. 20150622005JC).

\section{REFERENCES}

1. Kaneto H, Miyatsuka T, Shiraiwa T, Yamamoto K, Kato K, Fujitani Y, Matsuoka TA. Crucial role of PDX-1 in pancreas development, beta-cell differentiation, and induction of surrogate beta-cells. Curr Med Chem. 2007; 14:1745-1752.

2. Jonsson J, Carlsson L, Edlund T, Edlund H. Insulinpromoter-factor 1 is required for pancreas development in mice. Nature. 1994; 371:606-609.

3. Wright NM, Metzger DL, Borowitz SM, Clarke WL. Permanent neonatal diabetes mellitus and pancreatic exocrine insufficiency resulting from congenital pancreatic agenesis. Am J Dis Child. 1993; 147:607-609.

4. Stoffers DA, Zinkin NT, Stanojevic V, Clarke WL, Habener JF. Pancreatic agenesis attributable to a single nucleotide deletion in the human IPF1 gene coding sequence. Nat Genet. 1997; 15:106-110.

5. Toskes PP, Rosenbloom AL, Winter WE, Maclaren NK, Riley WJ, Andres J. Congenital pancreatic hypoplasia: a syndrome of exocrine and endocrine pancreatic insufficiency. J Pediatr. 1986; 109:465-468.

6. Howard CP, Go VL, Infante AJ, Gerich JE, Haymond MW. Long-term survival in a case of functional pancreatic agenesis. J Pediatr. 1980; 97:786-789.

7. Fan N, Lai L. Genetically modified pig models for human diseases. J Genet Genomics. 2013; 40:67-73. 
8. Prather RS, Lorson M, Ross JW, Whyte JJ, Walters E. Genetically engineered pig models for human diseases. Annu Rev Anim Biosci. 2013; 1:203-219.

9. Hani EH, Stoffers DA, Chèvre JC, Durand E, Stanojevic V, Dina C, Habener JF, Froguel P. Defective mutations in the insulin promoter factor-1 (IPF-1) gene in late-onset type 2 diabetes mellitus. J Clin Invest. 1999; 104:R41-48.

10. Yu D, Zhong Y, Li X, Li Y, Li X, Cao J, Fan Z, Fan H, Yuan L, Xu B, Yuan Y, Zhang H, Ji Z, et al. Generation of TALEN-mediated FH knockout rat model. Oncotarget. 2016; 7:61656-61669. https://doi.org/10.18632/ oncotarget.11429.

11. Kang JD, Kim SJ, Zhu HY, Jin L, Guo Q, Li XC, Zhang YC, Xing XX, Xuan MF, Zhang GL, Luo QR, Kim YS, Cui $\mathrm{CD}$, et al. Generation of cloned adult muscular pigs with myostatin gene mutation by genetic engineering. RSC Adv. 2017; 7:12541-12549.

12. Ke Q, Li W, Lai X, Chen H, Huang J, Kang Z, Li K, Ren J, Lin X, Zheng H, Huang W, Ma Y, Xu D, et al. TALENbased generation of a cynomolgus monkey disease model for human microcephaly. Cell Res. 2016; 26:1048-1061.

13. Kim H, Kim MS, Wee G, Lee CI, Kim H, Kim JS. Magnetic separation and antibiotics selection enable enrichment of cells with ZFN/TALEN-induced mutations. PLoS One. 2013; 8:e56476.

14. Kim H, Um E, Cho SR, Jung C, Kim H, Kim JS. Surrogate reporters for enrichment of cells with nuclease-induced mutations. Nat Methods. 2011; 8:941-943.

15. Tesson L, Usal C, Ménoret S, Leung E, Niles BJ, Remy S, Santiago Y, Vincent AI, Meng X, Zhang L, Gregory PD, Anegon I, Cost GJ. Knockout rats generated by embryo microinjection of TALENs. Nat Biotechnol. 2011; 29:695-696.

16. Sung YH, Baek IJ, Kim DH, Jeon J, Lee J, Lee K, Jeong D, Kim JS, Lee HW. Knockout mice created by TALENmediated gene targeting. Nat Biotechnol. 2013; 31:23-24.

17. Song J, Zhong J, Guo X, Chen Y, Zou Q, Huang J, Li X, Zhang Q, Jiang Z, Tang C, Yang H, Liu T, Li P, et al. Generation of RAG 1- and 2-deficient rabbits by embryo microinjection of TALENs. Cell Res. 2013; 23:1059-1062.

18. Carlson DF, Tan W, Lillico SG, Stverakova D, Proudfoot C, Christan M, Voytas DF, Long CR, Whitelaw CB, Fahrenkrug SC. Efficient TALEN-mediated gene knockout in livestock. Proc Natl Acad Sci U S A. 2012; 109:17382-17387.

19. Qian L, Tang M, Yang J, Wang Q, Cai C, Jiang S, Li H, Jiang K, Gao P, Ma D, Chen Y, An X, Li K, et al. Targeted mutations in myostatin by zinc-finger nucleases result in double-muscled phenotype in Meishan pigs. Sci Rep. 2015; $5: 14435$.
20. Wang K, Ouyang H, Xie Z, Yao C, Guo N, Li M, Jiao H, Pang D. Efficient generation of myostatin mutations in pigs using the CRISPR/Cas9 system. Sci Rep. 2015; 5:16623.

21. Hauschild J, Petersen B, Santiago Y, Queisser AL, Carnwath JW, Lucas-Hahn A, Zhang L, Meng X, Gregory PD, Schwinzer R, Cost GJ, Niemann H. Efficient generation of a biallelic knockout in pigs using zinc-finger nucleases. Proc Natl Acad Sci U S A. 2011; 108:12013-12017.

22. Fujimura T, Takahagi $Y$, Shigehisa $T$, Nagashima $H$, Miyagawa S, Shirakura R, Murakami H. Production of alpha 1,3-Galactosyltransferase gene-deficient pigs by somatic cell nuclear transfer: a novel selection method for gal alpha 1,3-Gal antigen-deficient cells. Mol Reprod Dev. 2008; 75:1372-1378.

23. Suzuki KT, Isoyama Y, Kashiwagi K, Sakuma T, Ochiai H, Sakamoto N, Furuno N, Kashiwagi A, Yamamoto T. High efficiency TALENs enable F0 functional analysis by targeted gene disruption in Xenopus laevis embryos. Biol Open. 2013; 2:448-452.

24. Hayflick L, Moorhead PS. The serial cultivation of human diploid cell strains. Exp Cell Res. 1961; 25:585-621.

25. Depinho RA. The age of cancer. Nature. 2000; 408:248-254.

26. $\mathrm{Wu} \mathrm{J}$, Izpisua Belmonte JC. Interspecies chimeric complementation for the generation of functional human tissues and organs in large animal hosts. Transgenic Res. 2016; 25:375-384.

27. Kobayashi T, Yamaguchi T, Hamanaka S, Kato-Itoh M, Yamazaki Y, Ibata M, Sato H, Lee YS, Usui J, Knisely AS, Hirabayashi M, Nakauchi H. Generation of rat pancreas in mouse by interspecific blastocyst injection of pluripotent stem cells. Cell. 2010; 142:787-799.

28. Wu J, Plateroluengo A, Sakurai M, Sugawara A, Gil MA, Yamauchi T, Suzuki K, Bogliotti YS, Cuello C, Morales VM, Okumura D, Luo J, Vilariño M, et al. Interspecies chimerism with mammalian pluripotent stem cells. Cell. 2017; 168:473-486.

29. Matsunari H, Nagashima H, Watanabe M, Umeyama K, Nakano K, Nagaya M, Kobayashi T, Yamaguchi T, Sumazaki R, Herzenberg LA, Nakauchi H. Blastocyst complementation generates exogenic pancreas in vivo in apancreatic cloned pigs. Proc Natl Acad Sci U S A. 2013; 110:4557-4562.

30. Yin XJ, Tani T, Yonemura I, Kawakami M, Miyamoto K, Hasegawa R, Kato Y, Tsunoda Y. Production of cloned pigs from adult somatic cells by chemically assisted removal of maternal chromosomes. Biol Reprod. 2002; 67:442-446.

31. Kim HJ, Lee HJ, Kim H, Cho SW, Kim JS. Targeted genome editing in human cells with zinc finger nucleases constructed via modular assembly. Genome Res. 2009; 19:1279-1288. 